\title{
Dieta de Myrmecophaga tridactyla (Pilosa: Myrmecophagidae] en pastizales y bosques del noreste de Santiago del Estero y su relación con las hormigas
}

\author{
Diet of Myrmecophaga tridactyla (Pilosa: Myrmecophagidae) in \\ pastures and forests of the northeast of Santiago del Estero and \\ its relationship with ants
}

D.0.I.: https://doi.org/10.30550/j.azl/2018.62.1/1

\author{
Andrea Fuster, Liliana Diodato, Jorge Homero Contreras \\ Instituto de Protección Vegetal (INPROVE), Facultad de Ciencias Forestales, Universidad Nacional de \\ Santiago Del Estero. Av. Belgrano 1912, (4200) Santiago del Estero, Argentina. \\ fusterandrea9@gmail.com
}

\begin{abstract}
> Resumen - El oso hormiguero gigante, Myrmecophaga tridactyla, se encuentra en estado de conservación vulnerable a causa de alteraciones en su hábitat. Conocer sus fuentes de alimentos, es decir, las especies de hormigas que son el componente más abundante de su dieta en las áreas semiáridas del Chaco, ayudará a generar estrategias para su conservación. Los objetivos de este estudio fueron verificar la presencia de $M$. trydactila en el noreste de Santiago del Estero, determinar la oferta alimenticia de hormigas en ambientes de pastizal y bosque e identificar la composición y abundancia de hormigas que conforman su dieta. Para comprobar la presencia del oso hormiguero gigante se recorrieron $22,4 \mathrm{~km}$ de transectas en ambos ambientes y se recolectaron evidencias de su presencia y heces. Para determinar la riqueza y abundancia de hormigas se efectuaron muestreos mediante trampas de caída. El análisis de heces indicó que la dieta comprendió diez géneros de hormigas, que corresponde al $50 \%$ de la riqueza de formícidos de la zona de estudio. Del total de hormigas identificadas en las heces el 95\% correspondió a Ectatomma sp. y Camponotus sp. Según los valores de riqueza, abundancia y similitud, la oferta de hormigas para la alimentación de $M$. tridactyla es similar en ambos ambientes. Esto sugiere que tanto el bosque como el pastizal constituyen ambientes propicios para la conservación de la especie en el noreste de Santiago del Estero. En general, las medidas de protección de hábitat para la conservación de la especie en la región deberían considerar la importancia de ambos ambientes.

Palabras clave: oso hormiguero gigante, distribución geográfica de mamíferos, hormigas, conservación biológica, biología alimentaria.
\end{abstract}

- Abstract - The giant anteater Myrmecophaga tridactyla is in a vulnerable state of conservation due to alterations occurred in its habitat. Knowing its food sources, namely the ant species which are the most abundant component of its diet in the Chaco semiarid areas will help generate strategies for its conservation. This study aimed to prove the presence of the $M$. tridactyla in the north-west part of Santiago del Estero, determine food offer of ant in

\footnotetext{
$>$ Ref. bibliográfica: Fuster, A., Diodato, L., Contreras, J. H. (2018). Dieta de Myrmecophaga tridactyla (Pilosa: Myrmecophagidae) en pastizales y bosques del noreste de Santiago del Estero y su relación con las hormigas. Acta zoológica lilloana 62 [1]: 1-9.

> Recibido: 28/11/17 - Aceptado: 09/05/18

> URL de la revista: http://actazoologica.lillo.org.ar

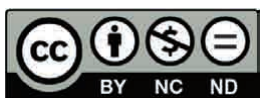

> Algunos derechos reservados. Esta obra está bajo una Licencia Creative Commons Atribución - No Comercial - Sin Obra Derivada 4.0 Internacional.
} 
grassland and forest environments in addition to identify composition and abundance of the ants making up its diet. To attest the presence of the giant anteater, $22.4 \mathrm{~km}$ of transects were traversed in both environments by collecting evidences and feces. To determine ant richness and abundance, samplings were done using pitfall tramps. Feces analysis showed that the diet comprised ten ant genres that equals 50\% of the formicide richness in the area under study. From the total of the ants identified in feces, 95\% belonged to Ectatomma sp. and Camponotus sp. According to the values of richness, abundance and similarity obtained, the ant offer for the $M$. tridactyla's diet is similar in both environments. All the above suggests that both the forest and the grasslands in the northest of Santiago del Estero are propitious for conserving the species. In general, the importance of these two environments should be considered by the policies protecting the conservation of the specie in this area.

Keywords: giant anteater, mammals geographical distribution, ants, biological conservation, food biology.

\section{INTRODUCCIÓN}

El oso hormiguero gigante, Myrmecophaga tridactyla Linnaeus, 1758, por su tamaño, es uno de los mamíferos más representativos de la fauna de América del Sur (Rodríguez-Mahecha, Alberico, Trujillo, Jorgenson, 2006). La vulnerabilidad de la especie ante presiones antrópicas originó su extinción en distintas regiones de América (Superina, Miranda, Abba, 2010). Actualmente, M. tridactyla se encuentra en el listado de especies amenazadas de la Unión Internacional para la Conservación de la Naturaleza (UICN) (Miranda, Bertassoni, Abba, 2014). La combinación de características biológicas de la especie, tales como la baja tasa reproductiva, la alta especialización de dieta, la baja capacidad de fuga, $\mathrm{y}$ los periodos de cuidados de cría prolongados, junto con la degradación del hábitat, incendios naturales y ocasionales atropellamientos por automóviles inciden en su supervivencia (Fonseca y Aguiar, 2004).

La distribución geográfica del oso hormiguero gigante comprende desde Belice y Guatemala (Emmons y Feer, 1997) hasta el norte de Argentina (Parera, 2002). En Argentina su área de distribución actual comprende las provincias de Misiones, Formosa, centro y norte de Chaco, este de Salta y norte de Santiago del Estero (Fonseca y Aguiar, 2004). Antiguamente su distribución llegaba hasta los $31^{\circ} \mathrm{S}$ de latitud (Parera, 2002; Superina et al., 2010), sin embargo, debido a las intensas modificaciones en su hábitat, los paralelos $29^{\circ} \mathrm{S}$ y $27^{\circ} \mathrm{S}$ se mencionan actualmente como límites más australes (Fon- seca y Aguiar, 2004; Pérez Jimeno y Amaya, 2009). Varios autores citan la presencia de M. trydactila en Santiago del Estero (Chébez, 1994; Díaz y Bárquez, 2002; Parera, 2002; Fonseca y Aguiar, 2004; Vizcaíno, Abba, García Esponda, 2006; Pautasso, 2007; Jimeno y Amaya, 2009), con registros en los departamentos Copo, Pellegrini, Taboada, Jiménez, Avellaneda y Belgrano, en un rango latitudinal de $25^{\circ} 50^{\prime} \mathrm{S}$ a $28^{\circ} 52^{\prime} \mathrm{S}$ y longitudinal de $62^{\circ} 46^{\prime} \mathrm{W}$ a $64^{\circ} 16^{\prime} \mathrm{W}$ (Pérez Jimeno y Amaya, 2009).

Myrmecophaga tridactyla se alimenta exclusivamente de hormigas y termitas (Montgomery y Lubin, 1977; Montgomery, 1985). Sin embargo, en ambientes semiáridos del Chaco, donde las hormigas son más abundantes que las termitas (Diodato, 2005), el oso hormiguero gigante se alimenta casi exclusivamente de ellas (Gallo et al., 2017). Existen varios estudios sobre la especialización de la dieta de M. tridactyla (Medri, Mourão, Harada, 2003; Braga, 2010; Sandoval-Gomez, Ramírez-Chaves, Marín, 2012; Braga, Souza, Batista, Dos Santos Lima, 2014; Gallo et al., 2017). En estos trabajos se identificó que la dieta del oso está compuesta principalmente por las hormigas de los géneros: Camponotus Mayr, Solenopsis Westwood, Odontomachus Latreille, Labidus Jurine, Linepithema Mayr, Acromyrmex Mayr, Atta Fabricius, Gnamptogenys Roger y Ectatomma Smith, F. El hábitat del oso hormiguero gigante es amplio y diverso. Está presente en bosques tropicales lluviosos y secos, y áreas de sabanas y pastizales abiertos (Rodríguez-Mahecha et al., 2006). A su vez, Formicidae es un grupo de 
insectos estrechamente vinculado a la fisonomía vegetal, y su biodiversidad varía según el tipo de ambiente (Basset, 2001; Gotelli y Ellison, 2002; Basset, Hammond, Barrios, Holloway, Miller, 2003; Ribas, Schoereder, Pic, Soares, 2003; Vasconcelos, Leite, Vilhena, Lima, Magnusson, 2008; Fuster, 2013). En consecuencia, se espera que la composición de la dieta de $M$. trydactila varíe y dependa de la disponibilidad de hormigas (Medri et al., 2003).

En la provincia de Santiago del Estero, en las últimas décadas, los paisajes naturales se modificaron, alterándose la composición, el área de cobertura y otros parámetros ecológicos de las comunidades vegetales (Boletta, Ravelo, Planchuelo, Grilli, 2006; Boletta y Ravelo, 2009). Para el desarrollo de estrategias de conservación de las poblaciones del oso hormiguero gigante a largo plazo, se requiere integrar el conocimiento sobre comportamiento, requerimientos alimenticos y hábitats con información local y específica (Rojano, Miranda, Ávila, 2015). Los objetivos de este estudio fueron verificar la presencia de $M$. trydactila en el noreste de Santiago del Estero, determinar la oferta alimenticia de hormigas en ambientes de pastizal y bosque; e identificar la composición y abundancia de hormigas que conforman su dieta.

\section{MATERIALES Y MÉTODOS}

El área de estudio se encuentra en el noreste de la provincia de Santiago del Estero (SE) en Otumpa, departamento Moreno (2718'51.80"S- 62¹0'8.90”W), en la región del Chaco semiárido argentino (Cabrera, 1976). El clima de la zona de estudio es cálido, con una temperatura media mensual de $28^{\circ} \mathrm{C}$ en el mes más caliente (enero), y 16,3 ${ }^{\circ} \mathrm{C}$ para el mes más frío (julio). Los valores extremos de temperatura registrados en la región son $47^{\circ} \mathrm{C} \mathrm{y}-10{ }^{\circ} \mathrm{C}$. La precipitación anual varía entre 600 y $800 \mathrm{~mm}$, concentrada en los meses estivales (Boletta et al., 2006; Boletta y Ravelo, 2009). La vegetación del Chaco semiárido se compone de bosques xerófitos, estepas y sabanas resultantes de incendios y desmontes (Cabrera, 1976). En el área de estudio se distinguen dos fisonomías vegetales: (1) bosque alto de Schinopsis lorentzii (Griseb.) Engl y Aspidosperma quebracho-blanco Schltr. y las especies secundarias Prosopis nigra Griseb, Acacia praecox Griseb., Ziziphus mistol Griseb. y Geoffroea decorticans (Gill. ex Hook. \& Arn.) Burkart; (2) pastizal, con pies arbóreos aislados de $S$. lorentzii, acompañados de un estrato vegetativo compuesto de arbustos y diversas herbáceas.

Para determinar la presencia del oso hormiguero gigante en el bosque y el pastizal, se realizaron cuatro transectos lineales de $100 \mathrm{~m}$ por ambiente, separados por $500 \mathrm{~m}$ como mínimo. En cada transecto se observaron signos de la presencia de $M$. trydactila, tales como: huellas, excavaciones en nidos de hormigas, heces y observación directa. Como registro secundario se consideraron las informaciones de moradores locales sobre la presencia del oso hormiguero gigante. Los datos se recopilaron semanalmente durante siete meses, de junio a diciembre de 2011.

Las heces recolectadas de $M$. trydactila se analizaron en el laboratorio. Se separaron los restos de hormigas, especialmente la cápsula cefálica, que es la parte del exoesqueleto más conservado y de fácil reconocimiento; y fueron identificadas a nivel de género. Este proceso se realizó bajo lupa binocular estereoscópica, lavando el material con alcohol al $70 \%$.

Para determinar la oferta alimenticia de formícidos, en febrero de 2012, se marcaron tres transectos de $100 \mathrm{~m}$ de longitud separados entre sí por $100 \mathrm{~m}$, en cada ambiente estudiado. En cada transecto se colocaron 10 trampas de caída que estuvieron activas por un periodo de 48 hs (Bestelmeyer et al., 2000). Las hormigas capturadas fueron identificadas a nivel específico. Se calcularon los valores de riqueza y abundancia de hormigas en cada ambiente y se determinó la diferencia significativa entre ellos mediante un análisis de Kruskal-Wallis (Infostat versión 2011; Di Rienzo et al., 2011). Los valores de similitud entre el ensamble de hormigas del bosque y del pastizal fueron obtenidos por el índice de Jaccard, calculado con el programa 
EstimateS v.8.2 (Colwell, 2009). Para analizar la variación en composición entre los ensambles de hormigas, se aplicó un análisis de similitud (ANOSIM) unidireccional con 9999 permutaciones y nivel de confianza del 95\%, mediante el programa PAST v. $2.17 \mathrm{~b}$ (Hammer, Harper, Ryan, 2001).

\section{RESULTADOS}

Se verificó la presencia de $M$. trydactila en los sitios estudiados, por registros de signos de actividad de la especie. Se recolectaron tres heces en el bosque y dos en el pastizal. Se observaron 12 huellas, ocho en el bosque y cuatro en el pastizal. De las cuatro excavaciones registradas, una se encontró en el bosque y tres en el pastizal.

A partir del muestreo de heces, se obtuvieron 1.687 cabezas de hormigas, pertenecientes a cinco subfamilias y 10 géneros. La dieta incluyó especímenes correspondientes a las subfamilias Myrmicinae, Formicinae, Ectatomminae, Ponerinae y Pseudomyrmecinae. Ectatomma y Camponotus fueron los géneros más abundantes (Tabla 1), representando el 95\% de las hormigas registradas. Otros géneros identificados en las heces fueron Odontomachus y Gnamptogenys, hormigas de gran tamaño corporal. En menor cantidad se registraron Pheidole Westwood; Solenopsis; Trachymyrmex Forel; Acromyrmex Mayr; Pogonomyrmex Mayr y Pseudomyrmex Lund.

A partir de los muestreos de hormigas, se encontraron seis subfamilias (Myrmicinae, Dolichoderinae, Formicinae, Ectatomminae, Ponerinae y Pseudomyrmecinae), 20 géneros y 35 especies y se recolectaron 3.337 individuos (Tabla 2). En el bosque y en el pastizal se detectaron las seis subfamilias. En el bosque se recolectaron 1.897 hormigas identificadas en 19 géneros y 27 especies. En tanto, en el pastizal se recolectaron 1.440 hormigas pertenecientes a 19 géneros y 31 especies. No se encontraron diferencias significativas entre ambientes para la riqueza específica $(\mathrm{H}=0,05 ; \mathrm{P}>0,9999)$ y la abundancia $(\mathrm{H}$ $=0,43 ; \mathrm{P}=0,700)$ de hormigas. En ambos ambientes, los géneros Forelius Emery, Camponotus, Ectatomma y Pheidole fueron domi- nantes en número de individuos. Forelius y Camponotus fueron los más abundantes en el bosque, mientras que Ectatomma y Pheidole lo fueron en el pastizal.

La similitud de los ensambles de hormigas del bosque y del pastizal fue del 65\%, lo que indica un bajo recambio de especies. El análisis de similitud (ANOSIM) mostró que los ensambles de hormigas fueron similares entre ambientes $(R=-0,24 ; P=0,7976)$.

\section{DISCUSIÓN}

El registro de la presencia de $M$. trydactila en el departamento Moreno, Santiago del Estero, se encuentra en el rango geográfico indicado por otros autores (Chébez, 1994; Díaz y Bárquez, 2002; Parera, 2002; Fonseca y Aguiar, 2004; Vizcaíno et al., 2006; Pautasso, 2007; Pérez Jimeno y Amaya, 2009). Con este nuevo registro de la presencia del oso hormiguero gigante a los $27^{\circ}$ $\mathrm{S}$ se comprueba que aún se conserva a esta latitud, a pesar de las transformaciones actuales del paisaje, en contraposición con lo que mencionan algunos autores (Superina et al., 2010). Esta información es de gran valor para conservar la especie en el área geográfica estudiada. Además, se debe resaltar que esta área presenta la mayor ocurrencia de focos de incendio en Santiago del Estero (Zerda, 2009), una de las principales causas de muerte de individuos del oso hormiguero gigante (Fonseca y Aguiar, 2004; Superina et al., 2010), por lo cual se requiere implementar estrategias de manejo del fuego.

El consumo de hormigas de Ectatomma sp. y Camponotus sp. por M. trydactila coinciden con observaciones realizadas por otros autores (Carvalho, 1966; Montgomery, 1985; Medri et al., 2003; Braga, 2010, Sandoval-Gómez et al., 2012; Braga et al., 2014; Gallo et al., 2017) en diferentes regiones de América del Sur; quienes mencionan la preferencia por Camponotus sp. y que el consumo de Ectatomma sp. es menos frecuente. La preferencia por Camponotus sp. fue observada inicialmente por Montgomery (1985), quien indicó que el oso hormiguero gigante estaría adaptado para alimentarse de estas 
Tabla 1. Subfamilias y géneros de hormigas identificados en las heces de Myrmecophaga tridactyla, en el bosque y en pastizal de Otumpa, departamento Moreno, Santiago del Estero.

\begin{tabular}{|c|c|c|c|}
\hline Formicidae & Heces & Bosque & Pastizal \\
\hline \multicolumn{4}{|l|}{ Myrmicinae } \\
\hline Acromyrmex & 11 & 11 & 12 \\
\hline Cyphomyrmex & 0 & 4 & 8 \\
\hline Kalathomyrmex & 0 & 2 & 1 \\
\hline Trachymyrmex & 1 & 1 & 6 \\
\hline Cephalotes & 0 & 0 & 5 \\
\hline Crematogaster & 0 & 10 & 95 \\
\hline Pheidole & 11 & 73 & 137 \\
\hline Pogonomyrmex & 1 & 3 & 4 \\
\hline Solenopsis & 3 & 1 & 3 \\
\hline Wasmannia & 0 & 29 & 3 \\
\hline \multicolumn{4}{|l|}{ Formicinae } \\
\hline Brachymyrmex & 0 & 18 & 16 \\
\hline Nylanderia & 0 & 79 & 15 \\
\hline Camponotus & 641 & 433 & 263 \\
\hline \multicolumn{4}{|l|}{ Dolichoderinae } \\
\hline Dorymyrmex & 0 & 1 & 2 \\
\hline Forelius & 0 & 1109 & 589 \\
\hline Gracilidris & 0 & 9 & 8 \\
\hline \multicolumn{4}{|l|}{ Pseudomyrmecinae } \\
\hline Pseudomyrmex & 1 & 18 & 36 \\
\hline \multicolumn{4}{|l|}{ Ectatomminae } \\
\hline Ectatomma & 957 & 92 & 236 \\
\hline Gnamptogenys & 4 & 2 & 0 \\
\hline \multicolumn{4}{|l|}{ Ponerinae } \\
\hline Odontomachus & 57 & 2 & 1 \\
\hline Total de subfamilias & 5 & 6 & 6 \\
\hline Total de géneros & 10 & 19 & 19 \\
\hline
\end{tabular}

hormigas. Probablemente esta preferencia en la alimentación se justifique por el mayor tamaño corporal de los individuos de Ectatomma sp. y Camponotus sp. De esta manera, consumiendo pocos individuos se incorpora un mayor aporte nutricional (Sandoval-Gómez et al., 2012). Por otro lado, ambos géneros de hormigas son muy abundantes en la región (Fuster, 2013), por lo que se infiere que $M$. trydactila se alimenta del recurso de mayor disponibilidad. En Colombia, Sandoval-Gómez et al. (2012) observaron que $M$. trydactila se alimentaba con más frecuencia de géneros con especies de tamaños más pequeños como Solenopsis sp., en contraposición a lo observado en este estudio. Sin embargo, coinciden que ello se debería a la
Tabla 2. Especies de hormigas identificadas en el bosque y en pastizal de Otumpa, departamento Moreno, Santiago del Estero.

\begin{tabular}{lccc}
\hline Formicidae & Bosque & Pastizal & Total \\
\hline Myrmicinae & 2 & 5 & 7 \\
Acromyrmex hispidus Santschi & 2 & 2 & 6 \\
\hline Acromyrmex striatus Roger & 4 & 5 & 10 \\
\hline Acromyrmex lundi Guérin-Méneville & 5 & 8 & 12 \\
\hline Cyphomyrmex rimosus (Spinola) & 4 & 1 & 3 \\
\hline Kalathomyrmex emeryi (Forel) & 2 & 6 & 7 \\
\hline Trachymyrmex pruinosus Emery & 1 & 1 & 1 \\
\hline Cephalotes eduarduli (Forel) & 0 & 4 & 4 \\
\hline Cephalotes pusillus (Klug) & 0 & 91 & 101 \\
\hline Crematogaster sp. & 10 & 4 & 4 \\
\hline Crematogaster crinosa (Mayr) & 0 & 39 & 72 \\
\hline Pheidole jelskii (Mayr) & 33 & 1 & 1 \\
\hline Pheidole aberrans (Mayr) & 0 & 24 & 31 \\
\hline Pheidole sp. & 7 & 73 & 106 \\
\hline Pheidole radozkowskii (Mayr) & 33 & 0 & 3 \\
\hline Pogonomyrmex naegelii Forell & 3 & 4 & 4 \\
\hline Pogonomyrmex cunicularius Mayr & 0 & 2 & 3 \\
\hline Solenopsis sp. 1 & 1 & 1 & 1 \\
\hline Solenopsis sp. 2 & 0 & 3 & 32 \\
\hline Wasmannia auropuntata (Roger) & 29 & & \\
\hline Formicinae & & & \\
\hline
\end{tabular}

\section{Formicinae}

\begin{tabular}{lccc} 
Brachymyrmex patagonicus Mayr & 18 & 16 & 34 \\
\hline Nylanderia fulva (Mayr) & 79 & 15 & 94 \\
\hline Camponotus substitutos Emery & 138 & 205 & 343 \\
\hline Camponotus rufipes (Fabricius) & 12 & 48 & 60 \\
\hline Camponotus leydigi Forel & 1 & 0 & 1 \\
\hline Camponotus renggeri Emery & 54 & 6 & 60 \\
\hline Camponotus punctulatus Mayr & 228 & 4 & 232
\end{tabular}

\section{Dolichoderinae}

Dorymyrmex carettei Forel Dorymyrmex exsanguis Forel Dorymyrmex bituber Santschi Forelius nigriventris Forel

\begin{tabular}{llll}
\hline Grasilidris pombero Wild \& Cuezzo & 9 & 8 & 17
\end{tabular}

Pseudomimecinae

Pseudomyrmex denticollis Emery $\quad 18 \quad 36 \quad 54$

Ectatomminae

Ectatomma bruneunn Smith F. $\quad 92 \quad 236 \quad 328$

\begin{tabular}{llll}
\hline Gnamptogenys sulcata (Smith F.) & 2 & 0 & 2
\end{tabular}

\section{Ponerinae}

Odontomachus chelifer (Latreille) $\quad 2 \quad 1 \quad 3$

\begin{tabular}{llll}
\hline Total & 1897 & 1440 & 3337 \\
\hline
\end{tabular}

abundancia de estas hormigas en el ambiente, $\mathrm{y}$ al fácil acceso a sus nidos.

El tercer género en orden de abundancia en las heces analizadas fue Odontomachus sp., que no resultó abundante en el bosque ni en el pastizal. Esto se explicaría por el 
tamaño corporal grande (Kusnezov, 1978), y por el comportamiento poco agresivo de las obreras cuando los nidos son perturbados (Deyrup, Trager, Carlin, 1985). Por lo cual se infiere que el tamaño y la no agresividad de estas hormigas facilitan la captura.

Estas observaciones sugieren que $M$. trydactila elegiría sus presas según accesibilidad, abundancia, tamaño corporal y estrategias de defensa débiles, características por las cuales las termitas no estén incluidas con frecuencia en su dieta en esta región. Probablemente esto se deba a la diferencia en riqueza y abundancia entre hormigas y termitas (Diodato, 2005). Para la provincia se citan 120 especie de hormigas (Fuster, 2013) mientras que para toda la región del Chaco semiárido solo se reportaron 18 especies de termitas (Cuezzo, 2005). Varios autores coinciden que la dieta de $M$. tridactyla consiste principalmente de hormigas terrestres (Montgomery, 1985), y que el consumo de termitas se produce en bajos porcentajes (Medri et al., 2003; Braga, 2010, SandovalGómez et al., 2012; Braga et al., 2014; Gallo et al., 2017). Si bien el oso hormiguero está especializado en incluir en su dieta a ambos grupos de insectos, las hormigas constituyen la mayor oferta disponible. Cunha et al. (2015) destacan la existencia de defensas químicas en termitas como un criterio de selección en la alimentación del oso hormiguero. Así, termites Cornitermes Wasmann con defensas químicas menos irritantes que otros géneros son las más frecuentemente consumidas.

En el bosque y el pastizal estudiados la mayor oferta de hormigas para la alimentación del oso hormiguero gigante estaría constituida por los géneros Forelius, Ectatomma y Camponotus. Sin embargo, en las heces analizadas no se encontraron restos de Forelius. Existe un único registro del consumo del género en Brasil (Braga, 2010; Braga et al., 2014). La baja presencia de Forelius en la dieta del oso hormiguero gigante podría explicarse por la dificultad en la captura, ya que estas hormigas se desplazan con movimientos rápidos (Cuezzo, 2000) y lo obligarían a largos periodos de asedio, lo que implicaría un rédito menor en términos de energía y mayor riesgo de depredación. Myrmecophaga tridactyla permanece frente a los nidos de hormigas por periodos cortos, probablemente para evitar las estrategias defensivas de las hormigas y no quedar mucho tiempo expuestos a sus propios depredadores (Montgomery, 1985; Redford, 1985).

Varios autores (Braga, 2010; SandovalGómez et al., 2012; Braga et al., 2014; Gallo et al., 2017) determinaron que el oso hormiguero gigante consume hormigas cortadoras de hojas de los géneros Atta y Acromyrmex. En el presente estudio, se observó que los restos de Acromyrmex sp. encontrados en las heces recolectadas correspondieron solamente a obreras menores. Probablemente, esto se deba a la presencia de las grandes espinas torácicas en las castas de obreras mayores (soldados), lo que dificultaría la ingesta para el mamífero. En este estudio, las hormigas del género Atta no fueron identificadas en las heces recolectadas, tampoco fueron identificadas en el bosque o en el pastizal, aunque son hormigas comunes en la región (Fuster, 2013). Otros géneros presentes en los sitios muestreados y que constituirían la oferta alimenticia son: Cephalotes Latreille, Cyphomyrmex Mayr, Kalathomyrmex Forel, Wasmannia Forel, Brachymyrmex Mayr, Nylanderia Emery, Gracilidris Wild \& Cuezzo y Dorymyrmex Mayr. Sin embargo, no se registró su consumo en la alimentación del oso hormiguero gigante. Las especies de $\mathrm{Ce}$ phalotes anidan en los árboles (De Andrade y Baroni Urbani, 1999; Brown, 2000), por lo que no estarían en igual disponibilidad que las especies que anidan en el suelo. Si bien M. tridactyla tiene la capacidad de trepar árboles, parece preferir alimentarse a nivel del suelo (Young, Coelho, Wieloch, 2003). En coincidencia con este trabajo, los géneros Cyphomyrmex, Kalathomyrmex y Gracilidris no están registrados en la dieta de $M$. tridactyla en ambientes naturales (Braga, 2010; Sandoval-Gómez et al., 2012; Braga et al., 2014; Gallo et al., 2017). Los géneros Dorymyrmex, Wasmannia, Brachymyrmex y Nylanderia se registraron en el consumo del oso hormiguero gigante, únicamente en 
ambientes altamente perturbados en Brasil, donde estas hormigas abundan (Möcklinghoff, 2008).

El alto valor de similitud de la composición de los ensambles de hormigas entre el bosque y el pastizal; y los valores semejantes de riqueza y abundancia sugieren que la oferta de hormigas para la alimentación de $M$. tridactyla es el mismo en ambos ambientes. A pesar de ello, el bosque es el ambiente más ventajoso para el oso hormiguero gigante pues también encuentra refugio, mientras que en el pastizal solo tiene mayor acceso a los nidos de hormigas; coincidiendo con lo determinado por otros autores (Camilo-Alves y Mourão, 2006; Mourão y Medri, 2007; Di Blanco, Pérez, Di Bitetti, 2015). De esta forma se explicaría por qué el oso hormiguero gigante está presente en ambos ambientes de Santiago del Estero.

Este trabajo muestra que $M$. trydactila se encuentra en Santiago del Estero desplazándose tanto en el bosque como en el pastizal, con similar oferta alimenticia. Esta información de base debería tenerse en cuenta en la gestión de áreas protegidas y programas de conservación de la fauna.

\section{AGRADECIMIENTOS}

Los autores agradecen a la Secretaría de Ciencia y Técnica de la Universidad Nacional de Santiago del Estero por la financiación del presente trabajo. Se agradece a los revisores anónimos que con sus sugerencias y observaciones mejoraron la versión final.

\section{FINANCIAMIENTO}

El presente trabajo fue financiado mediante el proyecto de investigación "Biodiversidad, conservación y uso sustentable del recurso fauna en la región del Chaco semiárido", (2 Etapa), Código 23B/118, Secretaría de Ciencia y Técnica de la Universidad Nacional de Santiago del Estero.

\section{PARTICIPACIÓN}

En este trabajo la primera autora contribuyó en la identificación del material bio- lógico obtenido de los muestreos, procesamiento de datos y escritura del manuscrito. La segunda autora participó en el procesamiento de datos y escritura, y el tercer autor realizó los muestreos y también colaboró en la escritura.

\section{CONFLICTOS DE INTERÉS}

En el presente trabajo no existen conflictos de interés entre los autores o con terceros.

\section{REFERENCIAS}

Basset Y. (2001). Invertebrates in the canopy of tropical rain forests: how much do we really know? Plant Ecology, 153, 87107.

Basset Y., Hammond, P. M., Barrios, H., Holloway, J. D., Miller, S. E. (2003). Vertical stratification of arthropod assemblages. EnArthropods of tropical forests. Spatio-temporal dynamics and resource use in the canopy (17-27). Cambridge: Cambridge University Press.

Bestelmeyer B. T., Agosti, D., Alonso, L. E, Brandao, C. R. F., Brown Jr., W. L., Delabie, J. H. C., Silvestre, R. (2000). Field techniques for study of grounddwelling ants: an overview, description and evaluation. EnAnts: standard methods for measuring and monitoring biodiversity (122-144). Washington, D.C.: Smithsonian Institution Press.

Boletta P. E., Ravelo, A. C., Planchuelo, A. M., Grilli, M. (2006). Assessing deforestation in the Argentine Chaco. Forest Ecology and Management, 228, 108114.

Boletta P. E., Ravelo, A. C. (2009). La deforestación y el cambio del uso de la tierra en el Chaco seco: Problemática de la desertificación. EnCambios y problemas ambientales-Perspectivas para la acción. (153-176). Santiago del Estero, Argentina: Facultad de Ciencias Forestales-Universidad Nacional de Santiago del Estero.

Braga F. G. (2010). Ecologia e comportamento de tamanduá-bandeira Myrmecophaga tridactyla Linnaeus, 1758 no município de Jaguariaíva, Paraná. Tesis Doctoral, Curitiba, Paraná, Brazil. Universidade Federal do Paraná.

Braga F. G., Souza, N. J., Batista, A. C., Dos Santos Lima, P. P. (2014). Consumo de Formigas Cortadeiras por Tamanduá- 
Bandeira Myrmecophaga tridactyla (Linnaeus, 1758) em Plantios de Pinus spp. no Paraná, Brasil. Edentata, 15, 1-8.

Brown W. L. Jr. (2000). Diversity of ants. EnAnts: standard methods for measuring and monitoring biodiversity (45-79). Washington D. C.: Smithsonian Institution Press. .

Cabrera A. L. (1976). Regiones fitogeográficas argentinas. En Enciclopedia Argentina de Agricultura y Jardinería, II. (185]. Buenos Aires: ACME.

Camilo-Alves C de S. P., Mourão, G. (2006). Responses of a specialized insectivorous mammal (Myrmecophaga tridactyla) to variation in ambient temperature. Biotropica, 38, 52-56.

Carvalho J. C. (1966). Novos dados sobre a alimentação do tamanduá-bandeira (Myrmecophaga tridactyla Linnaeus, 1758), Edentata, Mammalia. The Anais da Academia Brasileira de Ciências, 38, 341-346.

Chébez J. C. (1994). Los que se van: especies argentinas en peligro. Buenos Aires: Editorial Albatros.

Colwell R. K. (2009). EstimateS: statistical estimation of species richness and shared species from samples. Version 8.2. hhtp//purl.oclc.org/estimates. Consulta: 4 de Julio de 2009.

Cuezzo F. (2000). Revisión del género Forelius (Hymenoptera: Formicidae: Dolichoderinae). Sociobiology, 35, 197-277.

Cuezzo C. [2005). Citas nuevas de Isoptera para el Chaco semiárido argentino. Revista de la Sociedad Entomológica Argentina, 64 (1-2), 106-108.

Cunha H. F., Carrijo, T. F., Prestes, A. C., Arruda, L. S., Rezende, P. B., Santos, T., Brandão, D. (2015). Food preference of giant anteater and collared anteater (Pilosa, Myrmecophagidae) regarding the termite defense strategies. Bioscience Journal,31, 234-241.

De Andrade M. L., Baroni Urbani C. (1999). Diversity and adaptation in the ant genus Cephalotes, past and present. Stuttgarter Beiträge zur Naturkunde, serie B. 271 .

Díaz M. M., Barquez, R. M. (2002). Los Mamíferos de Jujuy, Argentina. Buenos Aires: L.O.L.A.

Di Blanco Y. E., Pérez, I. J., Di Bitetti, M. S. [2015]. Habitat selection in reintroduced giant anteaters: the critical role of conservation areas. Journal of Mammalogy, 96,1024-1035.

Di Rienzo J. A., Casanove, F., Balzarini, M. G., González, L., Tablada, M., Robledo,
C. W. (2011). InfoStat versión 2011. Grupo InfoStat, FCA, Universidad Nacional de Córdoba, Argentina. http://www. infostat.com.ar. Consultada 5 de diciembre de 2011.

Deyrup M., Trager, J., Carlin, N. (1985). The genus Odontomachus in the southeastern United States. Entomological News, 96, 188-195.

Diodato L. (2005). Conservación de la diversidad de atrópodos (Insecta) en ambientes naturales del Chaco semiárido. En Santiago del Estero una mirada ambiental (207-224). Santiago del Estero: Facultad de Ciencias Forestales. UNSE.

Emmons L. H., Feer, F. [1997). Neotropical rainforest mammals: a field guide. Chicago: The University of Chicago Press.

Fonseca G. A. B., Aguiar, J. M. (2004). The 2004 Edentate species assessment workshop. Edentata, 6, 1-26.

Fuster, A. (2013). Diversidad de hormigas (Hymenoptera: Formicidae), en la provincia de Santiago del Estero, Chaco semiárido, Argentina. Tesis doctoral. Universidad Nacional de Tucumán.

Gallo J. A., Abba, A. M., Elizalde, L., Di Nucci, D., Ríos, T. A., Ezquiaga, M. C. (2017). First study on food habits of anteaters, Myrmecophaga tridactyla and Tamandua tetradactyla, at the southern limit of their distribution. Mammalia, 1-4.

Gotelli N. J., Ellison, A.M. (2002). Biogeography at a regional scale: determinants of ant species density in bogs and forests of New England. Ecology, 83, 16041609.

Hammer, Ø., Harper D. A. T., Ryan P. D. 2001. Past: Paleontological Statistics Software Package for Education and Data Analysis. Palaeontología Electrónica 4: 1-9.

Jimeno P. G., Amaya, L. L. (2009). Contribución al conocimiento de la distribución del oso hormiguero gigante (Myrmecophaga tridactyla) en Argentina. Edentata,8-12.

Kusnezov N. (1978). Hormigas argentinas: clave para su identificación. Fundación Miguel Lillo, Miscelánea, 61,1-146.

Medri I. M., Mourão, G. M., Harada, A. Y. [2003]. Dieta de tamanduá-bandeira [Myrmecophaga tridactyla) no Pantanal da Nhecolândia, Brasil. Edentata, 5, 2934.

Miranda F., Bertassoni, A., Abba, A. M. (2014). Myrmecophaga tridactyla. The IUCN Red List of Threatened Species, : e.T14224A47441961.

Montgomery G. G., Lubin, Y. B. (1977). Prey influences on movements of Neotropical 
anteaters. En Proceedings of the 1975 Predator Symposium Montana Forest and Conservation Experiment Station (103-131), Missoula, USA: University of Montana. .

Montgomery G. G. (1985). Movements, foraging and food habits of the four extant species of Neotropical vermilinguas (Mammalia: Myrmecophagidae). EnThe Evolution and Ecology of Armadillos, Sloths, and Vermilinguas (365-377). Washington, D.C.: Smithsonian Institution Press.

Möcklinghoff L. (2008). Social organization and habitat use of the giant anteater (Myrmecophaga tridactyla L., 1758) in timber plantations in Northern Brazil. Diploma Thesis. Faculty of Biology, Department of Animal Ecology and Tropical Biology, University of Würzburg, Germany.

Mourão G., Medri, I. M. (2007). Activity of a specialized insectivorous mammal (Myrmecophaga tridactyla] in the Pantanal of Brazil. Journal of Zoology, 271, 187192.

Parera A. (2002). Los mamíferos de la Argentina y la región austral de Sudamérica. Buenos Aires: Editorial El Ateneo.

Pautasso A. (2007). Mamíferos amenazados y casi amenazados en la colección del Museo Provincial de Ciencias Naturales «Florentino Ameghino», Santa Fe, Argentina. Mastozoología Neotropical, 14[1], 85-91.

Redford K. H. (1985). Feeding and food preferences in captive and wild giant anteater (Myrmecophaga tridactyla). Journal of Zoology, 205, 559-572.

Ribas C. R., Schoereder, J. H., Pic, M., Soares, S. M. (2003). Tree heterogeneity, resource availability, and larger scale processes regulating arboreal ant species richness. Austral Ecology,28, 305-314.

Rodríguez-Mahecha J., Alberico, M., Trujillo, F., Jorgenson, J. (2006). Libro rojo de los mamíferos de Colombia. Serie libros rojos de especies amenazadas de CoIombia. Bogotá: Conservación Internacional Colombia y Ministerio Ambiente, Vivienda y Desarrollo.

Rojano C., Miranda, L., Ávila, R. (2015). Densidad poblacional y biomasa del oso hormiguero gigante (Myrmecophaga tridactyla) en Pore, Casanare, Colombia. Revista Biodiversidad Neotropical, 5 (1), 64-70.

Sandoval-Gómez V. E., Ramírez-Chaves, H. E., Marín, D. (2012). Registros de hormigas y termitas presentes en la dieta de osos hormigueros (Mammalia: Myrmecophagidae) en tres localidades de Colombia. Edentata, 13, 1-9.

Superina M., Miranda, F., Abba, A. M. (2010). The 2010 anteater Red List assessment. Edentata, 11, 96-114.

Vasconcelos H. L., Leite, M. F., Vilhena, J. M. S, Lima, A. P., Magnusson, W. E. [2008). Ant diversity in an Amazonian savanna: relationship with vegetation structure, disturbance by fire, and dominant ants. Austral Ecology, 33, 221231.

Vizcaíno S. F., Abba, A. M., García Esponda, C. M. [2006]. Magnorden Xenarthra. EnMamíferos de Argentina: sistemática y distribución (47 - 48). Argentina: Sociedad Argentina para el Estudio de los Mamíferos (SAREM)..

Young R. J., Coelho, C. M., Wieloch, D. R. A. (2003). Note on the climbing abilities of giant anteaters, Myrmecophaga tridactyla (Xenarthra, Myrmecophagidae), Bolétim do Museu Biológia Mello Leitão, 15, 41-46.

Zerda, H. R. (2009). Distribución espacial y temporal del fuego en la provincia de Santiago del Estero. En: Cambios y problemas ambientales: perspectivas para la acción (275-294). Argentina: Edition Universidad Nacional de Santiago del Estero. 